\title{
Imitation in Japanese quail: The role of reinforcement of demonstrator responding
}

\author{
CHANA K. AKINS and THOMAS R. ZENTALL \\ University of Kentucky, Lexington, Kentucky
}

\begin{abstract}
Imitative learning has been difficult to demonstrate in animals, partly because techniques have not adequately ruled out alternative accounts based on motivational and perceptual mechanisms (Zentall, 1996). Recently, it has been proposed that differences in the effects of observation of two very different response topographies would rule out such artifactual, alternative accounts (Akins \& Zentall, 1996). In the present research, we confirmed that strong evidence for imitation can be found in Japanese quail, and that such imitation requires the imitator's observation of reinforced responding by the demonstrator. Thus, under the present conditions, it appears that an observer will imitate a demonstrated response only if it also observes the appetitive consequences of that response.
\end{abstract}

In the past decade, substantial progress has been made in the study of imitative behavior in animals (see Heyes, 1996). This progress can be attributed to the development of procedural techniques used to distinguish instances of imitation from other social influences on response matching (see Zentall, 1996). One of these procedural techniques is the two-action method suggested by Galef, Manzig, and Field (1986, based on an experiment by Dawson \& Foss, 1965). The two-action method involves exposing observers to a demonstrator performing a particular response with one of two different response topographies (e.g., in birds, stepping on a treadle with the foot vs. pecking the treadle with the beak). Imitation is evident when the observer acquires the treadle response using the same response topography as it has observed. The two-action method allows one to control for nonimitative social learning, such as learning facilitated by the inadvertent (confounded) manipulation of motivational or perceptual variables (e.g., the mere presence of a conspecific, and attention drawn to the manipulandum by demonstrator responding; see Zentall, 1996). This method has aided in the demonstration of imitative behavior in various species, including Japanese quail (Akins \& Zentall, 1996), pigeons (Zentall, Sutton, \& Sherburne, 1996), and budgerigars (Dawson \& Foss, 1965; Galef et al., 1986). Related methods, such as the bidirectional control procedure and the cross-target procedure have been useful for identifying imitation in rats (Heyes \& Dawson, 1990) and in human infants (Meltzoff \& Moore, 1977; see also Meltzoff, 1996, for a review). These findings suggest that imitation may be more prevalent in nature than it was once thought to be.

This research was supported by National Science Foundation Grants IBN-9511656 and BNS-9019080 to C. K. A. and T. R. Z., respectively, and National Institute of Mental Health Grant MH 45979 to T. R. Z. We thank Rebecca Elliot for help in training demonstrators and collecting test data. Correspondence concerning this article should be sent to $T$. $R$. Zentall, Department of Psychology, University of Kentucky, Lexington, Kentucky 40506-0044 (e-mail: zentall@pop.uky.edu).
Despite improved methodology, psychologists have been unable to agree on the mechanisms responsible for true imitative behavior. Although some researchers have attempted to account for imitation in terms of simple conditioning processes (e.g., Gewirtz, 1969; Miller \& Dollard, 1941), careful examination of the literature indicates that these simple accounts are insufficient (Zentall, 1996). At the other end of the cognitive continuum are others who view imitation as the capacity for cognitively mediated perspective taking (e.g., Piaget, 1945; Whiten, 1991). Given the high level of cognitive capacity needed to "imagine oneself in the place of another," this account, as well, appears unlikely to explain imitation, especially in nonprimate species such as pigeons and rats.

A better understanding of the mechanisms responsible for imitative learning may come from an experimental analysis of the conditions needed to produce it. In psychological research with children, positive reinforcement administered to a model (demonstrator), contingent on a particular behavior, increases the likelihood that an observer will copy that behavior (see, e.g., Bandura, 1962; Bandura, Ross, \& Ross, 1963; Bandura \& Walters, 1963; Berger, 1961; Kanfer, 1965; Kanfer \& Marston, 1963). This manipulation is referred to as vicarious reinforcement. Although there is evidence for the role of vicarious reinforcement in imitation, it is limited primarily to experiments with humans (Lewis \& Duncan, 1958; McBrearty, Marston, \& Kanfer, 1961) and rhesus monkeys (Darby \& Riopelle, 1959; Warden, Fjeld, \& Koch, 1940). For example, Groesbeck and Duerfeldt (1971) did not find a significant effect of the observation of reinforcement of demonstrator rats' behavior. Their observation conditions were less than ideal, however, because the observation chamber, which was behind the start alley of a Y maze, was relatively far removed from demonstrator reinforcement, which occurred at the end of one of the distant arms. Furthermore, rats are not noted for their visual acuity.

On the other hand, using rats and the bidirectional polepush procedure, Heyes, Jaldow, and Dawson (1994) did 
find evidence that demonstrator reinforcement affected the likelihood of observer imitation, but imitation of the directional pole-push response occurred only when the observed pole push was followed by a brief auditory signal that accompanied demonstrator reinforcement. Thus, observation of the response followed by demonstrator reinforcement was not sufficient for imitation to occur.

Our present purpose was to investigate the role of reinforcement of the demonstrator's response, in the imitative behavior of male Japanese quail, using the two-action method. A $2 \times 2$ factorial design was used involving as factors the demonstrator's response topography (i.e., stepping on or pecking at the treadle) and reinforcement (or its absence) of demonstrator responding. Observer quail were given visual access to a demonstrator that was either stepping on or pecking at a treadle, either for food or no food. The observers were then given access to the treadle, and the correspondence of response topography between observers and their respective demonstrator was examined. Finally, a control group was included which observed a demonstrator that was fed occasionally but that did not respond (i.e., neither pecked nor stepped). These observers provided a trial-and-error baseline against which to assess responding by the groups exposed to either of the two relevant behaviors.

\section{METHOD}

\section{Subjects}

Thirty-seven male Japanese quail, 5-20 months old, served as subjects. The birds were hatched and raised in the laboratory from eggs obtained from a randomly bred colony maintained in accordance with institutional guidelines at the University of Kentucky. The quail were kept in mixed-sex groups in brooders until $4-5$ weeks of age, when they were moved to individual wire mesh quail cages (GQF Manufacturing Co., Savannah, GA), where they were maintained at approximately $80 \%$ $85 \%$ of their free-feeding weights for the duration of the experiment. All of the subjects were experimentally naive to the apparatus and the conditions of the present experiment, but they had previously served as subjects in a taste conditioning experiment in which they were given normal or tainted food in an elongated and distinctly colored context. The colony room was maintained under a 16:8-h light:dark cycle.

\section{Apparatus}

The apparatus, illustrated in Figure 1, was the same as that used to test imitation in pigeons (Zentall et al., 1996) and that used in a previous study with Japanese quail (Akins \& Zentall, 1996). It consisted of two large modular test chambers (Coulbourn Instruments, Lehigh Valley, PA), each approximately $30.5 \mathrm{~cm}$ high, $25.5 \mathrm{~cm}$ wide, and $28.0 \mathrm{~cm}$ deep. One chamber, designated as the demonstrator's chamber, contained a treadle, a rectangular feeder opening, and a houselight, all mounted on a sidewall of the chamber (perpendicular to the wall separating the two chambers). The treadle, a modified rat lever, was a metal rectangle, approximately $3.8 \mathrm{~cm}$ square. The treadle was located about $1 \mathrm{~cm}$ from the common wall and was about $1.3 \mathrm{~cm}$ above the wire mesh floor. The feeder opening was located in the center of the sidewall of the chamber, and the shielded houselight was mounted near the ceiling above the feeder opening. Subjects were provided access to food (Southern States $40 \%$ Poultry Supplement) through the feeder opening whenever a feeder tray was raised and illuminated by the feeder light. A speaker, located near the ceiling in the demonstrator's chamber, provided white masking noise at about $72 \mathrm{~dB}$. The observer's chamber was similar to the demonstrator's chamber, except that it did not contain a treadle, houselight, or feeder, and the floor consisted of parallel metal rods.

\section{Procedure}

Five demonstrators were randomly selected to serve as stepping demonstrators $(n=2)$, pecking demonstrators ( $n=2)$, or a control demonstrator (neither pecking nor stepping, $n=1$ ). The 5 quail demonstrators were trained to eat from the feeder tray when it was raised. Following feeder training, 2 of the demonstrators were shaped to peck the treadle and 2 other demonstrators were shaped to step on the treadle. The schedule of reinforcement for the stepping and pecking demonstrators was then gradually extended until they were responding on a variable interval 120 -sec schedule (i.e., the first response after a variable interval that averaged $120 \mathrm{sec}$ was reinforced). This schedule ensured that the demonstrators would respond for at least $2.5 \mathrm{~min}$ during nonreinforced sessions when an observer was present. During training, 1 of the stepping demonstrators had to be replaced because it began to peck the treadle rather than step on the treadle.

During their last few days of training, prior to testing for observer imitation, the demonstrators received training with a "dummy" bird present in the observer's chamber. This was done to ensure that the presence of an observer would not disrupt the demonstrator's stepping or pecking performance. The remaining demonstrator was magazine trained and exposed to a "dummy" observer but was not trained to step on or peck the treadle (untrained responses to the treadle were not reinforced).

Each of the 32 observers was magazine trained in the demonstrator's chamber and was given 4-5 days of habituation in the observer's chamber, with no demonstrator present. Observers were randomly assigned to one of five groups: observed pecking reinforced $(n=7)$, observed

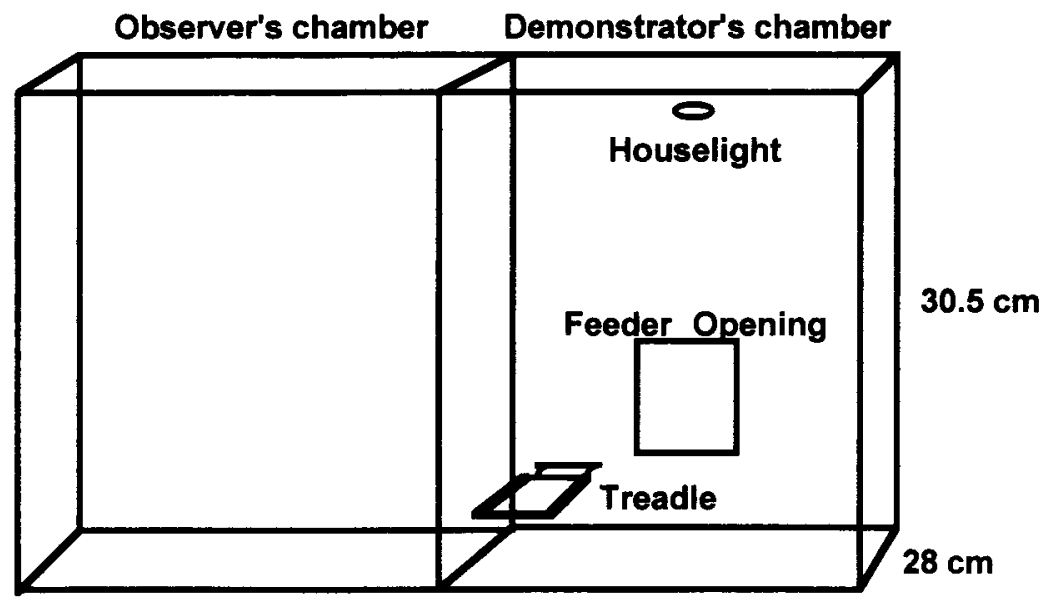

$25.5 \mathrm{~cm}$

Figure 1. A schematic diagram of the apparatus used in the present experiment. 
stepping reinforced $(n=6)$, observed pecking nonreinforced $(n=7)$, observed stepping nonreinforced $(n=6)$, or observed neither pecking nor stepping ( $n=6)$.

During each test session, an observer was placed in the observer's chamber while a demonstrator pecked the treadle or stepped on the treadle and received food following each response, received no food following each response, or did not respond but got fed (on the average, as often as the demonstrators that received food following each response) After $2.5 \mathrm{~min}$, the 1st demonstrator was removed and a 2 nd demonstrator was placed into the demonstrator's chamber. The second demonstrator performed the same response topography for an additional $2.5 \mathrm{~min}$ and experienced the same consequence for responding as did the Ist demonstrator. Two demonstrators were used for each observation session to permit a 5 -min observation session during which a high level of responding was maintained. All of the responding demonstrators pecked the treadle or stepped on the treadle at a rate no slower than one response per $10 \mathrm{sec}$. Control group observers received the same treatment as did the experimental observers, except that their demonstrator did not respond to the treadle but rather ate from the feeder tray when it was raised.

Immediately after the 5-min observation session, the demonstrator was removed, and the observer was placed into the demonstrator's chamber for a 10-min period, during which it was given the opportunity to respond. The frequency and topography of all observer responses were continuously recorded. Treadle responses were recorded automatically by means of a microswitch attached to the treadle. The two response topographies were easily distinguished by the experimenter because the posture of the quail when it pecked the treadle (head down, body away from the treadle) was quite different from its posture when it stepped on the treadle (head up, body over the treadle). Thus, there was no overlap in the response topographies.

\section{RESULTS}

Data from 5 of the observers that watched the responding demonstrators were not included in the analysis because these observers failed to respond more than four times during the $10-\mathrm{min}$ period in which they were given the opportunity to respond. Thus, data were analyzed for 5 observers exposed to a pecking and eating demonstrator, for 6 observers exposed to a stepping and eating demonstrator, for 5 observers exposed to a pecking but not eating demonstrator, for 5 observers exposed to a stepping but not eating demonstrator, and for 6 observers exposed to the control demonstrator.

For all analyses performed, the .05 level of statistical significance was adopted. Data are reported as the proportion of pecks, which were calculated as the frequency of pecking responses divided by the total frequency of pecking plus stepping responses.

The mean proportion of pecks for the observers during the 10-min test session is presented in Figure 2. A two-way analysis of variance (ANOVA) performed on the observers' responses, with response observed and demonstrator reinforcement versus nonreinforcement as variables, revealed a significant main effect of response observed $[F(1,17)=10.05]$. Thus, the mean proportion of pecking was higher for subjects that observed a demonstrator pecking ( $M=0.71, S E M=0.07)$ than for subjects that observed a demonstrator stepping $(M=0.30$, $S E M=0.11)$. The ANOVA also revealed a significant response observed $\times$ demonstrator reinforcement interaction $[F(1,17)=4.85]$. Simple main effects analyses were conducted on observers' responses to identify the

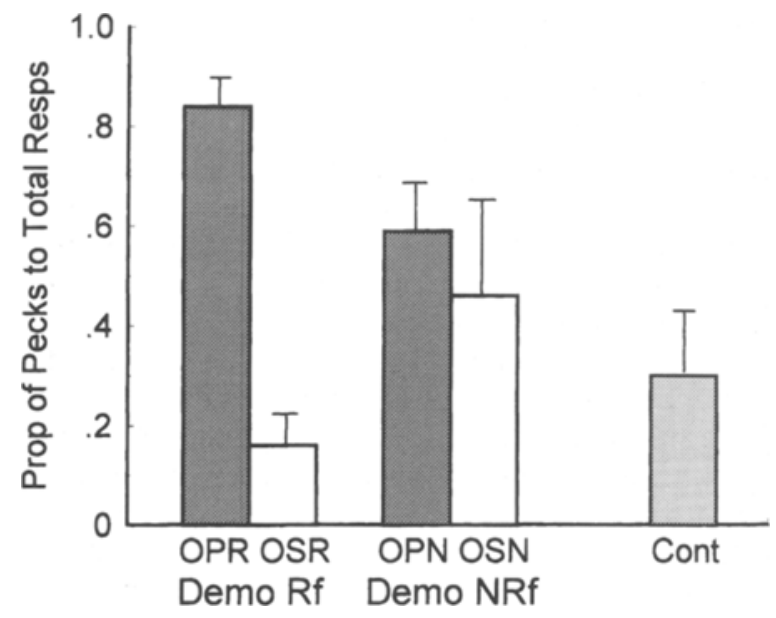

Figure 2. The mean ( $\pm S E M$ ) proportion of pecks made by quail that observed demonstrators receive reinforcement for pecking (OPR) or stepping (OSR), demonstrators receive no reinforcement for pecking (OPN) or stepping (OSN), or a demonstrator receive reinforcement for neither stepping nor pecking (Cont).

source of this interaction. The results indicated that subjects that observed reinforced pecking made a significantly higher proportion of their responses by pecking than did subjects that observed reinforced stepping $[F(1,17)=15.62]$. In contrast, there was no significant difference in the proportion of pecks made by subjects that observed nonreinforced pecking as opposed to nonreinforced stepping $[F(1,17)=0.50]$. Thus, imitative learning was found for quail that observed a demonstrator responding for food but not for quail that observed the response without reinforcement.

To compare the imitation groups and the baseline control group, two one-way ANOVAs were conducted. An ANOVA performed on the data from the observed pecking reinforced group, the observed stepping reinforced group, and the control group revealed a significant effect of response observed $[F(2,14)=12.92]$. The source of that effect can be attributed to a significant linear trend [i.e., the mean proportion of pecks for the control group $(M=0.30, S E M=0.13)$ fell between the mean proportion of pecks for the observed pecking reinforced group $(M=0.84, S E M=0.05)$ and the mean proportion of pecks for the observed stepping reinforced group $(M=0.16, S E M=0.07) ; F(1,14)=25.22]$.

The second ANOVA indicated that the proportion of pecks for the control group $(M=0.30, S E M=0.13)$, the observed stepping nonreinforced group $(M=0.46, S E M=$ 0.22 ), and the observed pecking nonreinforced group $(M=0.59, S E M=0.10)$ did not differ significantly from one another $[F(2,13)=0.45]$.

\section{DISCUSSION}

The present findings replicate and extend previous findings of true imitative behavior in Japanese quail. As in previous findings with quail (Akins \& Zentall, 1996), male quail that were provided visual access to 
pecking conspecifics pecked more than they stepped, and those that were provided visual access to stepping conspecifics stepped more than they pecked. This effect cannot be accounted for by stimulus enhancement (i.e., drawing attention to the moving treadle), because the twoaction method should result in similar stimulus-enhancing effects for both groups. Thus, the only difference between pecking demonstrators and stepping demonstrators occurred in their response topographies.

In contrast, observers that viewed demonstrators step on or peck the treadle in the absence of reinforcement did not perform response topographies similarly to their respective demonstrators. Instead, their pattern of responding did not differ reliably from that of the trial-anderror control group. The findings, therefore, indicate that the correspondence between observer and demonstrator response topography depends on observation of reinforcement of the demonstrator's responses This finding suggests that vicarious reinforcement is necessary for imitative behavior to occur in male Japanese quail.

As indicated earlier, the proportion of pecking responses made by the control group represents a baseline indicative of trial-and-error learning. The actual proportion of pecking responses will be affected by the relative difficulty of pecking as opposed to stepping. The size of the treadle, its height from the floor, and the effort required to make a response are all factors likely to affect this proportion. Pilot data suggested which values of these variables would result in approximately equal numbers (on the average) of trial-and-error pecks and steps. As can be seen in Figure 2, the proportion of pecks by the control group was comparable to that of both the observed stepping nonreinforced group and the observed pecking nonreinforced group and was different from that of the two groups that observed reinforced responding.

By what mechanism does the observation of demonstrator reinforcement result in imitation? Zentall (1996) has suggested that the pairing of a demonstrator's response (e.g., movement of a manipulandum) with a secondary reinforcer (the sight of food) might lead to a form of higher order conditioning that has been called observational conditioning. But this account relies on stimulus enhancement (attention drawn to the manipulandum), and, as already noted, when imitation has been found using the two-action method, stimulus enhancement cannot account for the different response topographies. Instead, rather than explaining imitation, it would appear that the observation of reinforced responding by the demonstrator acts as a catalyst or releaser of imitation. Such a mech anism can be described in two related ways. First, observation of demonstrator reinforcement (i.e., the sight of food) may provide the observer with the motivation to imitate. When similar effects of the observation of demonstrator reinforcement on imitation have been found in experiments involving human participants, however, they have been attributed to a more cognitive understanding by the observer of the consequences, to the demonstrator, of the demonstrated behavior (Bandura, 1962). But such an account relies on the ability of the observer to take the perspective of the demonstrator (Piaget, 1945), and it is quite unlikely that Japanese quail have this capacity. The fact that imitative learning can be demonstrated in very young children (Meltzoff, 1996) and animals suggests that perspective taking is not involved. Although there is no adequate account of the mechanisms responsible for imitation in species as putatively cognitively limited as Japanese quail, perhaps the reliable finding of imitation and the determination of conditions under which it can be elicited will stimulate the development of better models of its underlying mechanisms.

\section{REFERENCES}

AKIns, C. K., \& Zentall, T. R. (1996). Imitative learning in male Japanese quail (Coturnix japonica) using the two-action method. Journal of Comparative Psvchology, 110, 316-320.

BANDURA, A. (1962). Social learning though imitation. In M. R. Jones (Ed.), Nebraska Symposium on Motivation (pp. 211-269). Lincoln: University of Nebraska Press.
Bandura, A., Ross, D., \& Ross, S. A. (1963). Vicarious reinforcement and imitation. Journal of Abnormal \& Social Psychology, 67, 601-607.

BanduRA, A., \& WaLters, R. H. (1963). Social learning and personality development. New York: Holt, Rinehart \& Winston.

BERGER, S. M. (1961). Incidental learning through vicarious reinforcement. Psychological Reports, 9, 477-491.

Darby, C. L., \& Riopelle, A. J. (1959). Observational learning in the rhesus monkey. Journal of Comparative Physiological Psychology, 54, 94-98.

DAwson, B. V., \& Foss, B. M. (1965). Observational learning in budgerigars. Animal Behaviour, 13, 470-474.

Galef, B. G., Manzig, L. A., \& Field, R. M. (1986). Imitation learning in budgerigars: Dawson and Foss (1965) revisited. Behavioural Processes, 13, 191-202.

GEWIRTZ, J. L. (1969). Mechanisms of social learning: Some roles of stimulation and behavior in early human development. In D. A. Goslin (Ed.), Handbook of socialization theory and research (pp. 57-211). Chicago: Rand-McNally.

Groesbeck, R. W., \& Duerfeldt, P. H. (1971). Some relevant variables in observational learning of the rat. Psychonomic Science, 22, 41-43.

HEYES, C. M. (1996). Introduction: Identifying and defining imitation. In C. M. Heyes \& B. G. Galef (Eds.), Social learning in animals: The roots of culture (pp. 211-220). New York: Academic Press.

HEYES, C. M., \& DAWSON, G. R. (1990). A demonstration of observational learning using a bidirectional control. Quarterly Journal of Experimental Psychology, 42B, 59-71.

HeYes, C. M., JaL DOW, E., \& Dawson, G. R. (1994). Imitation in rats: Conditions of occurrence in a bidirectional control procedure. Learning \& Motivation, 25, 276-287.

KANFER, F. H. (1965). Vicarious human reinforcement: A glimpse into the black box. In L. Kramer (Ed.), Research in behavior modification (pp. 244-267). New York: Holt, Rinehart \& Winston.

KANFER, F. H., \& MARSTON, A. R. (1963). Human reinforcement: Vicarious and direct. Journal of Experimental Psychology, 65, 292-296.

LEWIS, D. J., \& DUNCAN, C. P. (1958). Vicarious experience and partial reinforcement. Journal of Abnormal Social Psychology, 57, 321-326.

McBrearty, J. F., Marston, A. R., \& Kanfer, F. H. (1961). Conditioning a verbal operant in a group setting: Direct vs. vicarious reinforcement. American Psychologist, 16, 425.

Meltzoff, A. N. (1996). The human infant as imitative generalist: A 20 -year progress report on infant imitation with implications for comparative psychology. In C. M. Heyes \& B. G. Galef (Eds.), Social learning in animals: The roots of culture (pp. 347-370). New York: Academic Press.

Meltzoff, A. N., \& MoORE, M. K. (1977). Imitation of facial and manual gestures by human neonates. Science, 198, 75-78.

Miller, N. E., \& Dollard, J. (1941). Social learning and imitation. New Haven, CT: Yale University Press.

PiageT, J. (1945). Play, dreams, and imitation in childhood. New York: Norton.

WARDEN, C. J., FJeld, H. A., \& KoCH, A. M. (1940). Imitative behavior in the cebus and rhesus monkeys. Journal of Genetic Psychology, $56,311-322$.

WHITEN, A. (1991). Natural theories of mind: Evolution, development and simulation of everyday mindreading. Cambridge, MA: Blackwell.

ZENTALL, T. R. (1996). An analysis of imitative learning in animals. In C. M. Heyes \& B. G. Galef (Eds.), Social learning in animals: The roots of culture (pp. 221-243). New York: Academic Press.

Zentall, T. R., Sutton, J. E., \& Sherburne, L. M. (1996). True imitative learning in pigeons. Psychological Science, 7, 343-346.

(Manuscript received August 28, 1997; revision accepted for publication February 13, 1998.) 